\title{
Application des commandes non linéaires pour la régulation en vitesse ou en position de la machine synchrone autopilotée
}

\author{
B. Le Pioufle ( $\left.{ }^{(}\right)$, G. Georgiou $\left({ }^{2}\right)$ et J.-P. Louis $\left({ }^{1}\right)$ \\ (') Laboratoire d'Electricité, Signaux et Robotique, L.E.Si.R., E.N.S. de Cachan, U.A. C.N.R.S. D1375, \\ 61 avenue du Président Wilson, 94235 Cachan Cedex, France \\ ( $\left.{ }^{2}\right)$ Laboratoire de Signaux et Systèmes, L.S.S., Ecole Supérieure d'Electricité, U.M. C.N.R.S. 14, Plateau du \\ Moulon, 91192 Gif-sur-Yvette Cedex, France
}

(Reçu le 18 décembre 1989, accepté le 15 février 1990)

\begin{abstract}
Résumé. - Dans cet article une méthode de l'automatique, la linéarisation totale par retour d'état, avec choix d'un comportement entrée-sortie, est appliquée à un actionneur électrique non linéaire, un servomoteur synchrone autopiloté. Cette solution est une alternative prometteuse aux stratégies classiques. Des exemples de dynamiques obtenues sur des commandes en vitesse ou en position, avec contrainte sur le courant d'axe direct, illustrent les possibilités de cette méthode.
\end{abstract}

\begin{abstract}
In this paper a method of modern control, total linearization by state-feedback, with choice of an input-output behaviour, is applied to a selfcontrolled servomotor. This solution may be a successful alternative to classical strategies. Some examples relative to speed or position control with constraint on the direct axis current show dynamical behaviours which illustrate the possibilities of this method.
\end{abstract}

\section{Introduction.}

Les servomoteurs synchrones se répandent de plus en plus comme actionneurs dans les industries automatisées où ils remplacent les moteurs à courant continu (cf. [5, 6]). Ils présentent sur ces derniers l'avantage d'avoir de meilleures performances (en terme de couple massique, par exemple) et de ne pas avoir de collecteur mécanique (ce collecteur pose des problèmes d'entretien et de comportement dans les environnements difficiles).

En revanche, ils sont plus exigeants. Le moteur à courant continu est alimenté par un convertisseur statique simple (un redresseur ou un hacheur), et une régulation de son courant d'induit permet de maîtriser le couple. Pour les moteurs synchrones, la fonction collecteur est réalisée par un ensemble électronique : un onduleur de puissance, une mesure de position et une commande adéquate des courants pour contrôler le couple.

Ainsi, en ce qui concerne les servomoteurs synchrones, un des principaux pôles de recherche porte sur le type de commande le plus approprié. Les stratégies traditionnelles consistent à asservir la machine en courant (donc en couple), en compensant les forces contre-électromotrices du moteur, et en découplant les équations différentielles régissant l'évolution des courants dans l'axe direct et dans l'axe en quadrature (cf. [10]). Le moteur est ensuite commandé en vitesse grâce à une boucle en cascade.

Ces stratégies classiques ignorent les non-linéarités propres aux machines synchrones que l'on observe immédiatement sur les équations d'un servomoteur synchrone. En outre, une analyse distincte des boucles de vitesse et de courant ne se justifie pas toujours, car les dynamiques de ces grandeurs sont parfois insuffisamment éloignées.

Rappelons les équations liant les tensions aux flux, dans le repère du rotor (variables de Park) :

$$
\left.\begin{array}{l}
V_{\mathrm{d}}=R_{\mathrm{s}} i_{\mathrm{d}}-p \Omega \phi_{\mathrm{q}}+\frac{\mathrm{d} \phi_{\mathrm{d}}}{\mathrm{d} t} \\
V_{\mathrm{q}}=R_{\mathrm{s}} i_{\mathrm{q}}+p \Omega \phi_{\mathrm{d}}+\frac{\mathrm{d} \phi_{\mathrm{q}}}{\mathrm{d} t}
\end{array}\right\}
$$

et celles liant les flux aux courants :

$$
\left.\begin{array}{l}
\phi_{\mathrm{d}}=L_{\mathrm{d}} i_{\mathrm{d}}+\Phi_{\mathrm{f}} \\
\phi_{\mathrm{q}}=L_{\mathrm{q}} i_{\mathrm{q}}
\end{array}\right\} .
$$

L'expression du couple en fonction du courant est :

$$
\begin{aligned}
C & =p\left[\left(L_{\mathrm{d}}-L_{\mathrm{q}}\right) i_{\mathrm{d}}+\Phi_{\mathrm{f}}\right] i_{\mathrm{q}} \\
& =J \frac{\mathrm{d} \Omega}{\mathrm{d} t}+f \Omega+C_{0}
\end{aligned}
$$


où :

$V_{\mathrm{d}}$ et $V_{\mathrm{q}}$ représentent respectivement les tensions statoriques de la machine ramenées dans les axes $\mathrm{D}$ et $\mathrm{Q}$ (direct et en quadrature).

$i_{\mathrm{d}} \quad$ est le courant, $L_{\mathrm{d}}$ est l'inductance, $\phi_{\mathrm{d}}$ est le flux dans l'axe $\mathrm{D}$.

$i_{\mathrm{q}}$ est le courant, $L_{\mathrm{q}}$ est l'inductance, $\phi_{\mathrm{q}}$ est le flux dans l'axe $Q$.

$R_{\mathrm{s}}$ représente la résistance statorique.

$\Omega$ est la vitesse du moteur.

$p \quad$ son nombre de paires de pôles.

$\Phi_{\mathrm{f}}$ est le flux généré par l'aimant permanent du rotor.

$J$ est l'inertie du système (moteur + charge).

$f$ le coefficient de frottement visqueux du système.

$C_{0}$ le couple résistant.

On obtient les courants $i_{\mathrm{d}}$ et $i_{\mathrm{q}}$ grâce à une reconstruction d'état à partir des courants mesurés dans les phases du moteur $i_{\mathrm{a}}, i_{\mathrm{b}}$ et $i_{\mathrm{c}}$, et de la position $\boldsymbol{\theta}$ :

$\left[\begin{array}{c}I_{\mathrm{d}} \\ I_{\mathrm{q}}\end{array}\right]=\sqrt{2 / 3}\left[\begin{array}{cc}\cos (p \theta) & \sin (p \theta) \\ -\sin (p \theta) & \cos (p \theta)\end{array}\right] \times$

$$
\times\left[\begin{array}{lll}
1 & -1 / 2 & -1 / 2 \\
0 & \sqrt{3 / 2} & -\sqrt{3 / 2}
\end{array}\right]\left[\begin{array}{c}
i_{\mathrm{a}} \\
i_{\mathrm{b}} \\
i_{\mathrm{c}}
\end{array}\right] .
$$

Les tensions simples du moteur sont calculées à l'aide des tensions $V_{\mathrm{d}}$ et $V_{\mathrm{q}}$ en appliquant la transformée inverse (cf. [5]) (c'est l'autopilotage).

Nous constatons dans ces équations la présence de deux types de non-linéarités : la multiplication par $\Omega$ dans les équations électriques, et le terme en $i_{\mathrm{d}}, i_{\mathrm{q}}$ dans l'expression du couple. Dans les régulations habituellement réalisées, le terme $\Omega$ qui intervient dans les équations électriques est considéré comme une constante (ou un paramètre lentement variable), et son effet (forces contre-electromotrices et couplage des équations dans l'axe direct et dans l'axe en quadrature) est, dans certaines études, compensé.

L'objet de ce travail est d'appliquer des méthodes de commande non linéaire développées récemment (cf. [7]), et donc de s'affranchir des approximations (découplage, linéarisation) habituellement employées.

Le contrôle doit être fait à l'aide des variables de Park.

\section{Présentation de la méthode non linéaire.}

Une présentation générale et exhaustive des méthodes de commande non linéaires serait rapidement lourde et exigerait la définition d'un appareil mathématique complexe. C'est pourquoi nous avons choisi un compromis en limitant le formalisme au strict minimum, en précisant à chaque fois les hypothèses nécessaires. Nous pourrons de cette manière appliquer immédiatement ces méthodes à la commande en vitesse, puis en position de la machine synchrone autopilotée. Pour un exposé plus détaillé sur les méthodes de commande non linéaires, on se rapportera aux articles cités en bibliographie $([4,1]$ pour les commandes en temps continu, [7, 9] pour les commandes sous échantillonnage, et [8] pour les commandes en temps discret).

2.1 MODÈLE DU MOTEUR. - Nous devons écrire les équations sous forme d'état, avec l'état :

$$
\mathbf{x}=\left[\begin{array}{l}
x_{1} \\
x_{2} \\
x_{3}
\end{array}\right], \quad x_{1}=i_{\mathrm{d}}, \quad x_{2}=i_{\mathrm{q}}, \quad x_{3}=\Omega
$$

et les entrées :

$$
\mathbf{u}=\left[\begin{array}{l}
u_{1} \\
u_{2}
\end{array}\right], \quad u_{1}=V_{\mathrm{d}}, \quad u_{2}=V_{\mathrm{q}} .
$$

On peut facilement réécrire les équations (1) et (2) sous la forme :

$$
\frac{\mathrm{d} x}{\mathrm{~d} t}=\mathbf{f}(\mathbf{x})+G \cdot \mathbf{u}
$$

avec :

$$
\mathbf{f}=\left\{\begin{array}{l}
f_{1}(\mathbf{x}) \\
f_{2}(\mathbf{x}) \\
f_{3}(\mathbf{x})
\end{array}\right\}, \quad G=\left\{\begin{array}{cc}
g_{1} & 0 \\
0 & g_{2} \\
0 & 0
\end{array}\right\}
$$

où :

$$
\begin{aligned}
& f_{1}(\mathbf{x})=a_{0} \cdot x_{1}+a_{1} \cdot x_{2} \cdot x_{3} \\
& f_{2}(\mathbf{x})=a_{0}^{\prime} \cdot x_{2}+a_{2} \cdot x_{1} \cdot x_{3}+a_{3} \cdot x_{3} \\
& f_{3}(\mathbf{x})=b_{1} \cdot x_{1} \cdot x_{2}+b_{2} \cdot x_{2}+b_{0} \cdot x_{3}-C_{\mathrm{r}} / J \\
& g_{1}=1 / L_{\mathrm{d}}, \quad g_{2}=1 / L_{\mathrm{q}}, \\
& \tau_{\mathrm{d}}=L_{\mathrm{d}} / R, \quad \tau_{\mathrm{q}}=L_{\mathrm{q}} / R, \quad \tau_{\mathrm{m}}=J / f \\
& a_{0}=-1 / \tau_{\mathrm{d}}, \quad a_{0}^{\prime}=-1 / \tau_{\mathrm{q}}, \quad a_{1}=p \cdot L_{\mathrm{q}} / L_{\mathrm{d}}, \\
& a_{2}=-p \cdot L_{\mathrm{d}} / L_{\mathrm{q}}, \quad a_{3}=p \cdot \Phi_{\mathrm{f}} / L_{q} \\
& b_{0}=-1 / \tau_{\mathrm{m}}, \quad b_{1}=p \cdot\left(L_{\mathrm{d}}-L_{\mathrm{q}}\right) / J, \\
& b_{2}=p \cdot \Phi, \Phi_{\mathrm{f}} / J .
\end{aligned}
$$

Nous pouvons voir sur la figure 1 une représentation schématique du moteur. Il y a deux commandes indépendantes $\left(u_{1}\right.$ et $\left.u_{2}\right)$, on pourra donc réguler deux variables.

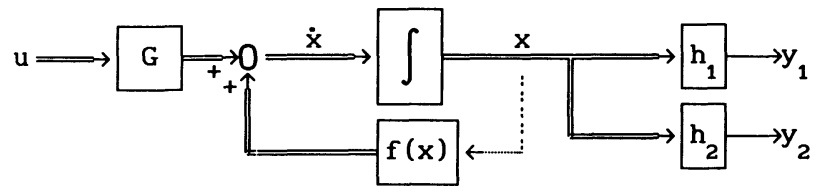

Fig. 1. - Représentation d'état du moteur.

[State representation of the motor.] 
Un point important à souligner est que le couple résistant $C_{\mathrm{r}}$ est une grandeur non mesurable. On peut seulement le supposer constant, ou lentement variable. Cela pose un problème pour l'implantation et nous en discuterons dans le paragraphe 3 .

2.2 PRINCIPE DE LA MÉTHOdE NON LINÉAIRE. La première étape de toute commande non linéaire consiste à définir quelles vont être les sorties du système, c'est-à-dire les variables à réguler.

Dans notre cas, ayant deux grandeurs de commandes $V_{\mathrm{d}}$ et $V_{\mathrm{q}}$, nous aurons donc la possibilité de régler indépendamment deux sorties $y_{1}$ et $y_{2}$.

Il s'agit ensuite d'écrire une équation différentielle matricielle liant une dérivée $n$-ième de la sortie $y_{1}$ et une dérivée $m$-ième de la sortie $y_{2}$ avec les commandes $V_{\mathrm{d}}$ et $V_{\mathrm{q}}$.

Dans la plupart des cas (et c'est le cas du moteur synchrone autopiloté), il est possible, grâce à un nombre suffisant de dérivations des sorties, d'obtenir :

$$
\left[\begin{array}{c}
\frac{\mathrm{d}^{n} y_{1}}{\mathrm{~d} t^{n}} \\
\frac{\mathrm{d}^{m} y_{2}}{\mathrm{~d} t^{m}}
\end{array}\right]=B_{0}+A_{0}\left[\begin{array}{c}
V_{\mathrm{d}} \\
V_{\mathrm{q}}
\end{array}\right]
$$

où $B_{0}$ est un vecteur de dimension 2 , et $A_{0}$, appelée matrice de découplage, est une matrice carrée d'ordre 2 que l'on veut inversible quel que soit le point de fonctionnement du moteur. Il faut remarquer que les composantes de $A_{0}$ et $B_{0}$ peuvent dépendre de l'état $X$.

De cette façon, en choisissant

$$
\left[\begin{array}{c}
V_{\mathrm{d}} \\
V_{\mathrm{q}}
\end{array}\right]=A_{0}^{-1}\left\{-B_{0}+\left[\begin{array}{l}
r_{1} \\
r_{2}
\end{array}\right]\right\},
$$

nous découplons le système car chaque entrée externe $r_{i}, i \in\{1,2\}$ agit seulement sur une des sorties $y_{i}$.

Nous obtenons le comportement suivant :

$$
\begin{aligned}
\frac{\mathrm{d}^{n} y_{1}}{\mathrm{~d} t^{n}} & =r_{1} \\
\frac{\mathrm{d}^{m} y_{2}}{\mathrm{~d} t^{m}} & =r_{2} .
\end{aligned}
$$

Pour imposer le régime statique $y_{1}=y_{1 \text { ref }}$ et $y_{2}=$

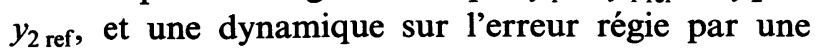
équation du type :

$$
\begin{aligned}
& \frac{\mathrm{d}^{n} e_{1}}{\mathrm{~d} t^{n}}=K_{n-1} \frac{\mathrm{d}^{n-1} e_{1}}{\mathrm{~d} t^{n-1}}+K_{n-2} \frac{\mathrm{d}^{n-2} e_{1}}{\mathrm{~d} t^{n-2}}+\cdots+K_{0} e_{1} \\
& \text { avec } e_{1}=y_{1 \text { ref }}-y_{1} \\
& \frac{\mathrm{d}^{m} e_{2}}{\mathrm{~d} t^{n}}=K_{m-1}^{\prime} \frac{\mathrm{d}^{m-1} e_{2}}{\mathrm{~d} t^{m-1}}+K_{m-2}^{\prime} \frac{\mathrm{d}^{m-2} e_{2}}{\mathrm{~d} t^{m-2}}+\cdots+K_{0}^{\prime} e_{2} \\
& \text { avec } e_{2}=y_{2 \text { ref }}-y_{2}
\end{aligned}
$$

les grandeurs $e_{1}$ et $e_{2}$ représentant respectivement les erreurs par rapport à la consigne sur les sorties $y_{1}$ et $y_{2}$, il faut choisir :

$$
\begin{aligned}
r_{1}= & K_{n-1} \frac{\mathrm{d}^{n-1} y_{1}}{\mathrm{~d} t^{n-1}}+K_{n-2} \frac{\mathrm{d}^{n-2} y_{1}}{\mathrm{~d} t^{n-2}}+\cdots+ \\
& +K_{0}\left(y_{1 \text { ref }}-y_{1}\right) \\
r_{2}= & K_{m-1}^{\prime} \frac{\mathrm{d}^{m-1} y_{2}}{\mathrm{~d} t^{m-1}}+K_{m-2}^{\prime} \frac{\mathrm{d}^{m-2} y_{2}}{\mathrm{~d} t^{m-2}}+\cdots+ \\
& +K_{0}^{\prime}\left(y_{2 \text { ref }}-y_{2}\right) .
\end{aligned}
$$

Le comportement du système est ainsi linéarisé. Les coefficients $K_{0}, K_{1}, \ldots, K_{n-1}, K_{0}^{\prime}, K_{1}^{\prime}, \ldots, K_{m-1}^{\prime}$, sont choisis en fonction des régimes statique et dynamique désirés.

Il suffit donc d'imposer le vecteur tensions de commande :

$$
\left[\begin{array}{c}
V_{\mathrm{d}} \\
V_{\mathrm{q}}
\end{array}\right]=A_{0}^{-1}\left[-B_{0}+\left[\begin{array}{c}
K_{n-1} \frac{\mathrm{d}^{n-1} y_{1}}{\mathrm{~d} t^{n-1}}+K_{n-2} \frac{\mathrm{d}^{n-2} y_{1}}{\mathrm{~d} t^{n-2}}+\cdots+K_{1} \frac{\mathrm{d} y_{1}}{\mathrm{~d} t}+K_{0}\left(y_{1 \text { ref }}-y_{1}\right) \\
K_{m-1}^{\prime} \frac{\mathrm{d}^{m-1} y_{2}}{\mathrm{~d} t^{m-1}}+K_{m-2}^{\prime} \frac{\mathrm{d}^{m-2} y_{2}}{\mathrm{~d} t^{m-2}}+\cdots+K_{1}^{\prime} \frac{\mathrm{d} y_{2}}{\mathrm{~d} t}+K_{0}^{\prime}\left(y_{2 \text { ref }}-y_{2}\right)
\end{array}\right]\right]
$$

Nous obtenons ainsi deux équations différentielles indépendantes régissant nos deux sorties. Nous pouvons régler le régime statique et placer les pôles à partir de ces deux équations.

3. Asservissement de vitesse utilisant les méthodes de commande non linéaires.

3.1 Commande CONTINUE. - Afin d'obtenir une linéarisation entrée-sortie totale, c'est-à-dire sans apparition d'une dynamique de zéros éventuellement instable (cf. [1, 4]), nous choisissons comme sorties, c'est-à-dire comme variables à contrôler, une combinaison linéaire des courants $i_{\mathrm{d}}$ et $i_{\mathrm{q}}$, ainsi que la vitesse.

$$
\begin{aligned}
& y_{1}=\alpha i_{\mathrm{d}}+\beta i_{\mathrm{q}} \\
& y_{2}=\Omega .
\end{aligned}
$$

Dérivons la sortie $y_{1}$ pour voir si une des commandes $V_{\mathrm{d}}$ où $V_{\mathrm{q}}$ apparaît :

$$
\frac{\mathrm{d} y_{1}}{\mathrm{~d} t}=\alpha f_{1}+\beta f_{2}+\left[\begin{array}{ll}
\alpha g_{1} & \beta g_{2}
\end{array}\right]\left[\begin{array}{l}
V_{\mathrm{d}} \\
V_{\mathrm{q}}
\end{array}\right]
$$


$g_{1}$ et $g_{2}$ sont toujours différents de 0 , en choisissant $\alpha$ ou $\beta$ non nul, on constate que l'action d'une des commandes apparaît effectivement.

Dérivons maintenant la deuxième sortie $y_{2}$ pour voir si une des commandes apparaît. Nous obtenons :

$$
\frac{\mathrm{d} y_{2}}{\mathrm{~d} t}=f_{3} .
$$

Nous voyons que la dérivée de la seconde sortie ne fait intervenir ni $V_{\mathrm{d}}$, ni $V_{\mathrm{q}}$. Il faut donc dériver une seconde fois cette sortie. Il faut alors faire une hypothèse sur le couple résistant $C_{0}$ dont on ne connaît pas la dérivée. On suppose ce couple constant par morceaux ou du moins très lentement variable. Nous avons pris cette hypothèse car elle est physiquement convenable. La dérivée du couple résistant par rapport au temps est donc nulle. Nous obtenons alors :

$$
\begin{aligned}
\frac{\mathrm{d}^{2} y_{2}}{\mathrm{~d} t^{2}}=f_{1} \frac{\partial f_{3}}{\partial x_{1}}+f_{2} \frac{\partial f_{3}}{\partial x_{2}}+f_{3} & \frac{\partial f_{3}}{\partial x_{3}}+ \\
& +g_{1} \frac{\partial f_{3}}{\partial x_{1}} V_{\mathrm{d}}+g_{2} \frac{\partial f_{3}}{\partial x_{2}} V_{\mathrm{q}}
\end{aligned}
$$

d'où :

$$
\begin{aligned}
\frac{\mathrm{d}^{2} y_{2}}{\mathrm{~d} t^{2}}=f_{1} b_{1} x_{2} & +f_{2}\left(b_{1} x_{1}+b_{2}\right)+f_{3} b_{0}+ \\
-+ & +\left[\begin{array}{lll}
g_{1} b_{1} x_{2} & g_{2}\left(b_{1} x_{\mathrm{I}}+b_{2}\right)
\end{array}\right]\left[\begin{array}{l}
V_{\mathrm{d}} \\
V_{\mathrm{q}}
\end{array}\right]
\end{aligned}
$$

Ici, les commandes apparaissent sous certaines conditions :

a) Si $L_{\mathrm{d}}=L_{\mathrm{q}}$ (machine à pôles lisses) :

$$
g_{1} b_{1} x_{2}=0 \text {, mais } g_{2}\left(b_{1} x_{1}+b_{2}\right)=g_{2} b_{2} \not \equiv 0,
$$

donc $V_{\mathrm{q}}$ apparaît.

b) Si $L_{\mathrm{d}} \not \equiv L_{\mathrm{q}}$ (machine à pôles saillants).

Il suffit d'avoir $x_{2} \not \equiv 0$ ou $x_{1} \not \equiv-b_{2} / b_{1}$.

La première condition n'est pas réalisée quel que soit le point de fonctionnement du moteur, car $x_{2}=i_{\mathrm{q}}$ peut être amené à changer de signe.

Il faut donc $x_{1} \not \equiv-b_{2} / b_{1}$

(c'est-à-dire $i_{\mathrm{d}} \not \equiv \frac{-\Phi_{\mathrm{f}}}{L_{\mathrm{d}}-L_{\mathrm{q}}}$ ).

Or avoir $\left(L_{\mathrm{d}}-L_{\mathrm{q}}\right) i_{\mathrm{d}}=-\Phi_{\mathrm{f}}$ revient à désexciter entièrement la machine en annulant le flux dû aux aimants rotoriques avec le flux de saillance. Ceci est irréalisable dans les machines que nous connaissons du fait de l'importance du courant alors nécessaire (cf. annexe).

Nous pouvons alors écrire l'équation différentielle :

$$
\left[\begin{array}{c}
\frac{\mathrm{d} y_{1}}{\mathrm{~d} t} \\
\frac{\mathrm{d}^{2} y_{2}}{\mathrm{~d} t^{2}}
\end{array}\right]=B_{0}+A_{0}\left[\begin{array}{c}
V_{\mathrm{d}} \\
V_{\mathrm{q}}
\end{array}\right]
$$

avec

$$
B_{0}=\left[\begin{array}{c}
\alpha f_{1}+\beta f_{2} \\
f_{1} b_{1} x_{2}+f_{2}\left(b_{1} x_{1}+b_{2}\right)+f_{3} b_{0}
\end{array}\right]
$$

et $\quad A_{0}=\left[\begin{array}{cc}\alpha g_{1} & \beta g_{2} \\ g_{1} b_{1} x_{2} & g_{2}\left(b_{1} x_{1}+b_{2}\right)\end{array}\right]$.

$\mathrm{Si}$ le déterminant de la matrice de découplage $A_{0}$ est non nul, ce dont nous discuterons plus bas, nous pouvons alors appliquer la loi de commande :

$$
\begin{aligned}
& {\left[\begin{array}{c}
V_{\mathrm{d}} \\
V_{\mathrm{q}}
\end{array}\right]=} \\
& =A_{0}^{-1}\left[-B_{0}+\left[\begin{array}{c}
K_{11}\left(y_{1 \mathrm{ref}}-y_{1}\right) \\
-K_{21} \frac{\mathrm{d} y_{2}}{\mathrm{~d} t}+K_{22}\left(y_{2 \mathrm{ref}}-y_{2}\right)
\end{array}\right]\right] \\
& =A_{0}^{-1}\left[-B_{0}+\left[\begin{array}{c}
K_{11}\left(y_{1 \mathrm{ref}}-y_{1}\right) \\
-K_{21} f_{3}+K_{22}\left(y_{2 \mathrm{ref}}-y_{2}\right)
\end{array}\right]\right] \text {. }
\end{aligned}
$$

Ceci nous permet d'imposer le régime statique $y_{1}=y_{1 \text { ref }}$ et $y_{2}=y_{2 \text { ref }}$, avec une dynamique sur l'erreur régie par une équation du type :

$$
\begin{array}{ll}
\frac{\mathrm{d} e_{1}}{\mathrm{~d} t}=K_{11} e_{1} \quad & \text { avec } e_{1}=y_{1 \text { ref }}-y_{1} \\
\frac{\mathrm{d}^{2} e_{2}}{\mathrm{~d} t^{2}}=K_{21} \frac{\mathrm{d} e_{2}}{\mathrm{~d} t}+K_{22} e_{2} & \text { avec } e_{2}=y_{2 \text { ref }}-y_{2} .
\end{array}
$$

Il est maintenant aisé de placer les pôles du système, en ajustant les coefficients $K_{11}, K_{21}$ et $K_{22}$.

Ayant obtenu la commande nécessaire à la linéarisation et au découplage de notre système, une remarque s'impose : dans le vecteur $B_{0}$ intervient $f_{3}$, faisant lui-même intervenir le couple de charge. Or, il est délicat de faire intervenir le couple de charge dans le calcul des tensions de commande du moteur, sachant que dans les systèmes électrotechniques classiques, cette grandeur n'est pas mesurée, ni facilement mesurable.

La solution retenue pour remédier à ce problème est de remplacer, dans l'expression des tensions de commande nécessaires, la fonction $f_{3}$ par une estimation de $\frac{\mathrm{d} \Omega}{\mathrm{d} t}$, grandeur pouvant être facilement calculée par le microprocesseur, en mesurant périodiquement la vitesse $\Omega$. A chaque période d'échantillonnage, le microprocesseur acquiert une nouvelle valeur de vitesse. Il lui suffit donc de retrancher à la valeur courante la valeur mesurée lors de la période d'échantillonnage précédente, puis de diviser la grandeur ainsi obtenue par la période d'échantillon- 
nage. Le microprocesseur calcule ainsi une valeur approchée de $\frac{\mathrm{d} \Omega}{\mathrm{d} t}$.

3.1.1 Discussion sur le déterminant de la matrice de découplage $A_{0}$. - Le déterminant de $A_{0}$ vaut :

$$
\begin{aligned}
\Delta=\frac{\alpha}{\tau_{\mathrm{d}} \tau_{\mathrm{q}}}\left[\frac{p\left(L_{\mathrm{d}}-L_{\mathrm{q}}\right)}{J} x_{1}\right. & \left.+\frac{p \Phi_{\mathrm{f}}}{J}\right]- \\
& -\frac{\beta}{\tau_{\mathrm{d}} \tau_{\mathrm{q}}}\left[\frac{p\left(L_{\mathrm{d}}-L_{\mathrm{q}}\right)}{J} x_{2}\right] .
\end{aligned}
$$

3.1.1.1 Cas où $L_{\mathrm{d}}=L_{\mathrm{q}}$ (machine à pôles lisses).

$$
\Delta=\frac{\alpha}{\tau_{\mathrm{d}} \tau_{\mathrm{q}}} \frac{p \Phi_{\mathrm{f}}}{J} \text {. }
$$

La matrice $A_{0}$ est alors toujours inversible si $\alpha \neq 0$.

3.1.1.2 Cas où $L_{\mathrm{d}} \not \equiv L_{\mathrm{q}}$ (machine à pôles saillants).

3.1.1.2.1 Cas où $\alpha=0$ et $\beta$. $\beta$. 0 : - Il est alors nécessaire d'avoir $i_{\mathrm{q}} \neq 0$, condition inacceptable, le courant $i_{\mathrm{q}}$ pouvant être amené à changer de signe. Ce cas doit donc être rejeté.

3.1.1.2.2 Cas où $\alpha \neq 0$ et $\beta=0$ - Il faut alors avoir $i_{\mathrm{d}} \not \equiv \frac{-\Phi_{\mathrm{f}}}{L_{\mathrm{d}}-L_{\mathrm{q}}}$, ce qui est une condition tout à fait acceptable puisqu'il suffit que $i_{\mathrm{d}}$ n'atteigne pas des valeurs difficilement accessibles (désexcitation totale du moteur) (cf. paragraphe 3.1.2).

3.1.1.2.3 Cas où $\alpha \neq 0$ et $\beta \not \equiv 0$ - Il faut alors avoir $\alpha i_{\mathrm{d}}-\beta i_{\mathrm{q}} \not \equiv \frac{-\Phi_{\mathrm{f}}}{L_{\mathrm{d}}-L_{\mathrm{q}}}$.

3.1.2 Etude du cas où $\alpha=1$ et $\beta=0$. - Nous avons décidé d'étudier plus particulièrement ce cas intéressant où nous régulons le courant $i_{\mathrm{d}}$ et la vitesse $\Omega$. En effet, si nous prenons alors comme références $x_{1 \text { ref }}=i_{\mathrm{d} \text { ref }}=0$ et $x_{3 \text { ref }}=\Omega_{\text {ref }}$, nous tra-

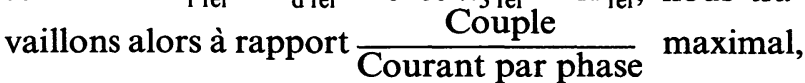
puisqu'il faut pour que ce rapport soit maximal que le courant soit dans l'axe q.

De plus, comme nous l'aivons vu dans le paragraphe précédent, le déterminant de la matrice $A_{0}$ est non nul, dans la mesure où $i_{\mathrm{d}}$ n'atteint pas $\frac{-\Phi_{\mathrm{f}}}{L_{\mathrm{d}}-L_{\mathrm{q}}}$ ce qui sera toujours le cas dans nos applications.

Dans ce cas le vecteur de commande $\left[\begin{array}{l}V_{\mathrm{d}} \\ V_{\mathrm{q}}\end{array}\right]$ vaut :

$V_{\mathrm{d}}=L_{\mathrm{d}} K_{11}\left(i_{\mathrm{d} \text { ref }}-i_{\mathrm{d}}\right)+R i_{\mathrm{d}}-p L_{\mathrm{q}} \Omega i_{\mathrm{q}}$

$$
\begin{aligned}
V_{\mathrm{q}}= & \frac{-\left(L_{\mathrm{d}}-L_{\mathrm{q}}\right) L_{\mathrm{q}}}{\left(L_{\mathrm{d}}-L_{\mathrm{q}}\right) i_{\mathrm{d}}+\Phi_{\mathrm{f}}} i_{\mathrm{q}}\left[K_{11}\left(i_{\mathrm{d} \text { ref }}-i_{\mathrm{d}}\right)\right]+R i_{\mathrm{q}} \\
& +p L_{\mathrm{d}} \Omega i_{\mathrm{d}}-p \Phi_{\mathrm{f}} \Omega+\frac{L_{\mathrm{q}} J}{p\left(L_{\mathrm{d}}-L_{\mathrm{q}}\right) i_{\mathrm{d}}+p \Phi_{\mathrm{f}}} \\
& \times\left[-K_{21} \frac{\mathrm{d} \Omega}{\mathrm{d} t}+K_{22}\left(\Omega_{\mathrm{ref}}-\Omega\right)+\frac{f}{J} \frac{\mathrm{d} \Omega}{\mathrm{d} t}\right] .
\end{aligned}
$$

Nous pouvons voir sur la figure 2 le schéma de principe de cet algorithme, et sur la figure 3 un essai indiciel en vitesse sur le moteur commandé par cet algorithme (temps de réponse à $95 \%$ de $45 \mathrm{~ms}$ ). Le moteur est supposé en charge (le couple résistant appliqué valant la charge maximale admissible $10 \mathrm{Nm}$ ). Les coefficients sont réglés de manière à ce que le courant dans le moteur ne dépasse pas le courant maximal admissible (30 A). Le courant $i_{\mathrm{d}}$ reste nul, et nous observons sur la figure 4 le pic de courant $i_{\mathrm{q}}$. Nous remarquons qu'en régime permanent $i_{\mathrm{q}}$ reste à $21 \mathrm{~A}$, ce qui permet de fournir le couple de charge.

Les résultats obtenus sont particulièrement prometteurs, si nous les comparons aux résultats des commandes classiques. En effet, les commandes classiques ne contrôlent pas les effets dus à la vitesse

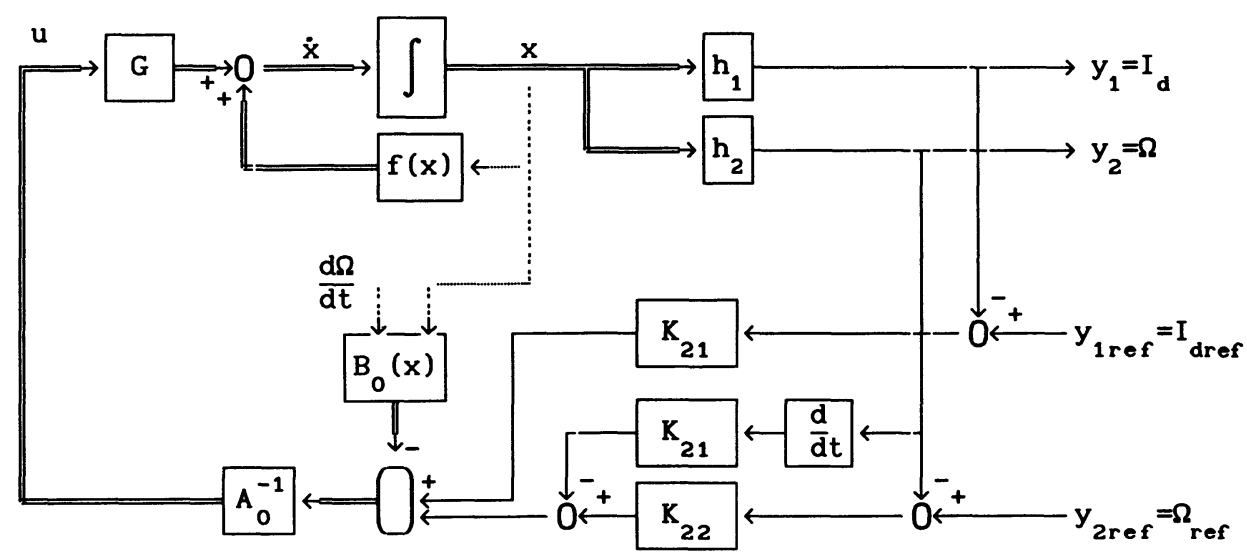

Fig. 2. - Schéma de principe de la commande non linéaire en vitesse.

[Scheme of the speed non linear control.] 


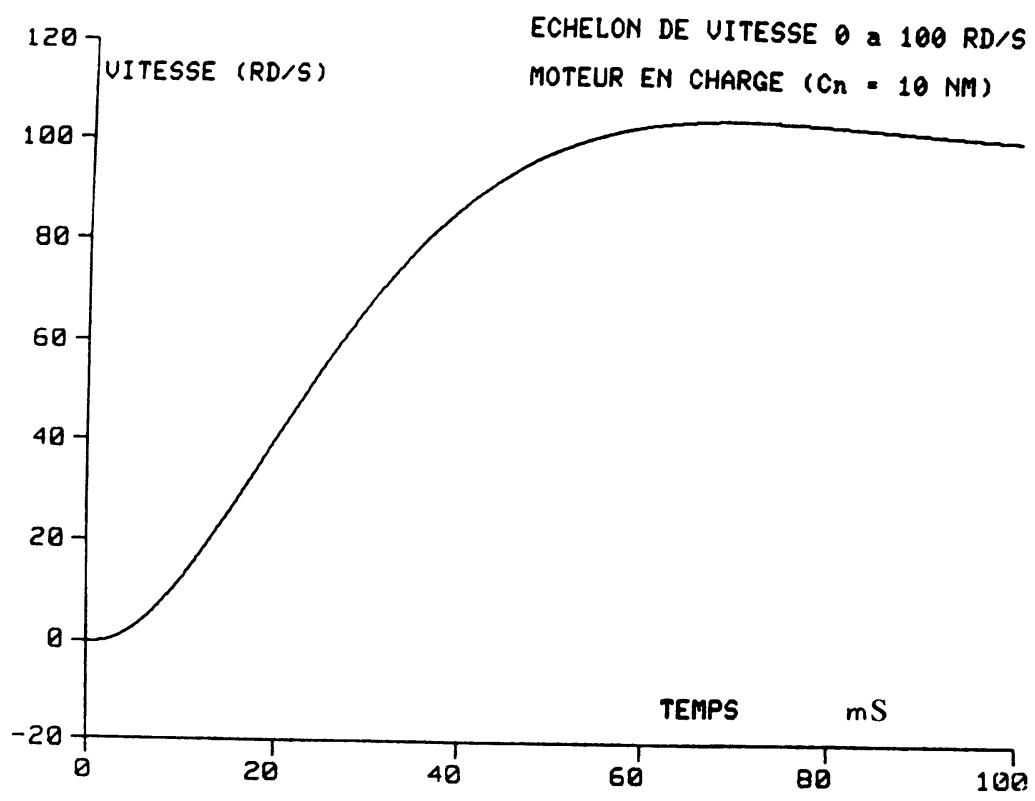

Fig. 3. - Commande non linéaire de vitesse. $\Omega=f(t)$.

[Speed non linear control. $\Omega=f(t)$.]

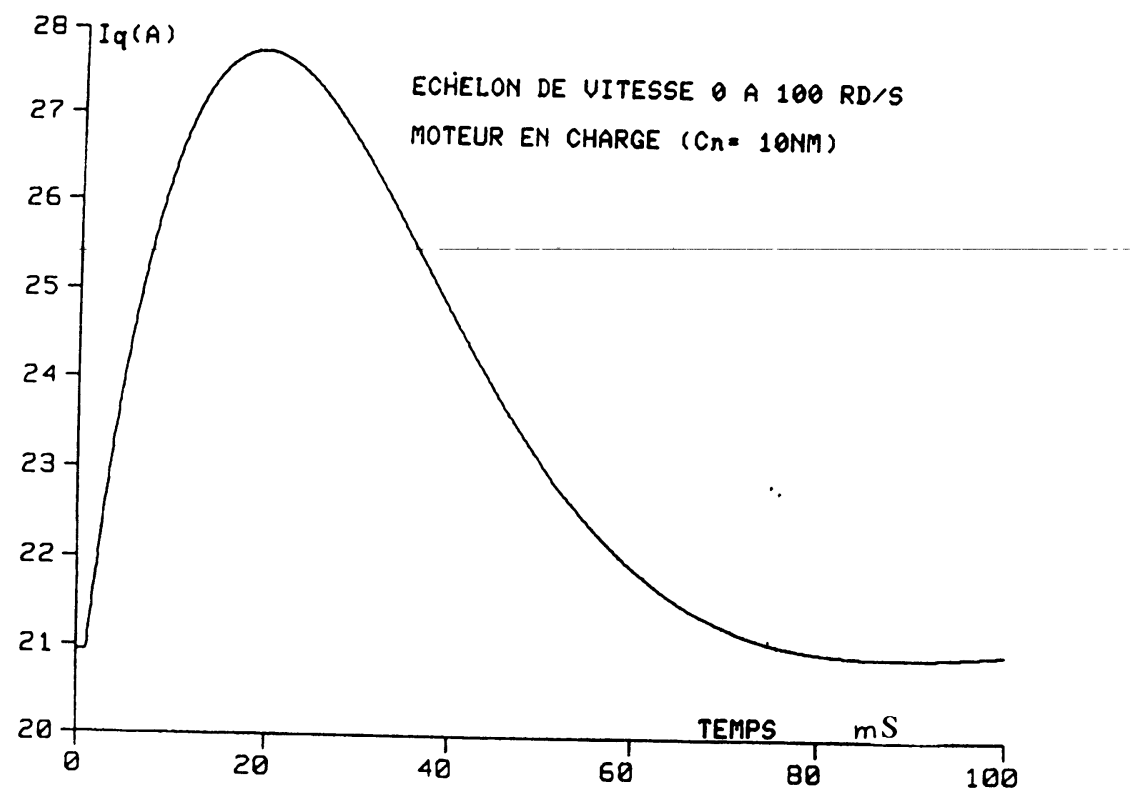

Fig. 4. - Commande non linéaire de vitesse. $I_{\mathrm{q}}=f(t)$.

[Speed non linear control. $I_{\mathrm{q}}=f(t)$.]

et l'accélération du moteur, ce qui fait que leur comportement est fortement altéré pour les conditions extrêmes de fonctionnement (on observe une forte diminution du couple moteur lorsque l'accélération est importante). Par contre, le correcteur non linéaire, linéarisant la réponse en vitesse grâce à une compensation directe des effets de $\Omega$ et de $\frac{\mathrm{d} \Omega}{\mathrm{d} t}$, permet d'obtenir le second ordre escompté pour la réponse indicielle en vitesse, quelle que soient les sollicitations en couple ou en vitesse.
3.2 COMMANDE NUMÉRIQUE. BLOQUEUR D'ORDRE 0 eT ReTARD DÛ AU TEMPS DE CAlCUl. - Pour l'implantation matérielle de la commande, nous utilisons un microprocesseur qui réalise l'autopilotage, la reconstruction d'état et l'algorithme de régulation. Les calculs sont donc discrétisés. De plus les mesures des courants et de la vitesse, proviennent d'un opérateur ayant la fonction blocage et convertion Analogique Numérique. Le bloqueur le plus simple que nous avons utilisé est un bloqueur d'ordre 0 . Comme conséquence de ces opérations, 
nous avons en premier lieu des tensions de commande $V_{\mathrm{d}}$ et $V_{\mathrm{q}}$ fixes pendant la période d'échantillonnage, du fait qu'elles sont calculées uniquement à partir des états mesurés.

De plus, il faut s'attarder sur le fait que l'algorithme numérique, qui fournit les tensions de consigne du moteur en fonction de l'état, nécessite un temps de calcul non nul, lié directement à la rapidité du microprocesseur utilisé et à la complexité de l'algorithme.

La commande est retardée par ce temps de calcul. Plusieurs stratégies sont alors possibles : la première consiste à utiliser le résultat de l'algorithme dès que son calcul est terminé, la seconde consistant à n'utiliser le résultat de l'algorithme qu'à la période d'échantillonnage suivante. Pour des raisons d'implantation plus aisée, nous avons opté pour la seconde méthode.

Nous pouvons voir sur la figure 5 la simulation de la commande non linéaire, incluant les phénomènes de discrétisation : bloqueur d'ordre 0 , et retard pur d'une période d'échantillonnage dû au temps de calcul du microprocesseur. Le temps de calcul étant estimé inférieur à $100 \mu \mathrm{s}$, performance parfaitement accessible à un processeur de signal classique. Nous voyons que le régulateur possède des propriétés statiques et dynamiques proches de celles obtenues pour le modèle continu, pour la période d'échantillonnage choisie $(100 \mu \mathrm{s})$. On observe que la réponse est néanmoins plus lente et plus amortie (temps de réponse à $95 \%$ de $92 \mathrm{~ms}$ ).

\section{Asservissement de position utilisant les méthodes} de commande non linéaires.

Nous voulons maintenant réguler la position du rotor, notée $\boldsymbol{\theta}$, de la machine, en utilisant les méthodes de commande non linéaire. Aux équations d'état (6) citées précédemment (cf. paragraphe 3), il nous faut maintenant ajouter l'équation (7) décrivant l'état $\theta$ :

$$
\begin{aligned}
& \dot{x}_{1}=f_{1}+g_{1} V_{\mathrm{d}} \\
& \dot{x}_{2}=f_{2}+g_{2} V_{\mathrm{q}} \\
& \dot{x}_{3}=f_{3} \\
& \dot{x}_{4}=x_{3} .
\end{aligned}
$$

Avec: $x_{1}=i_{\mathrm{d}}, x_{2}=i_{\mathrm{q}}, x_{3}=\Omega, x_{4}=\theta$.

Nous choisissons cette fois encore comme première sortie une combinaison linéaire de $i_{\mathrm{d}}$ et $i_{\mathrm{q}}$ :

$$
y_{1}=\alpha x_{1}+\beta x_{2} .
$$

Pour la deuxième sortie, nous prenons cette fois-ci la position $\theta$, puisque c'est elle que nous désirons réguler.

$$
y_{2}=x_{4} \text {. }
$$

Comme nous l'avons vu dans le paragraphe précédent, il suffit de dériver la première sortie une fois pour voir apparaître au moins une des tensions de commande :

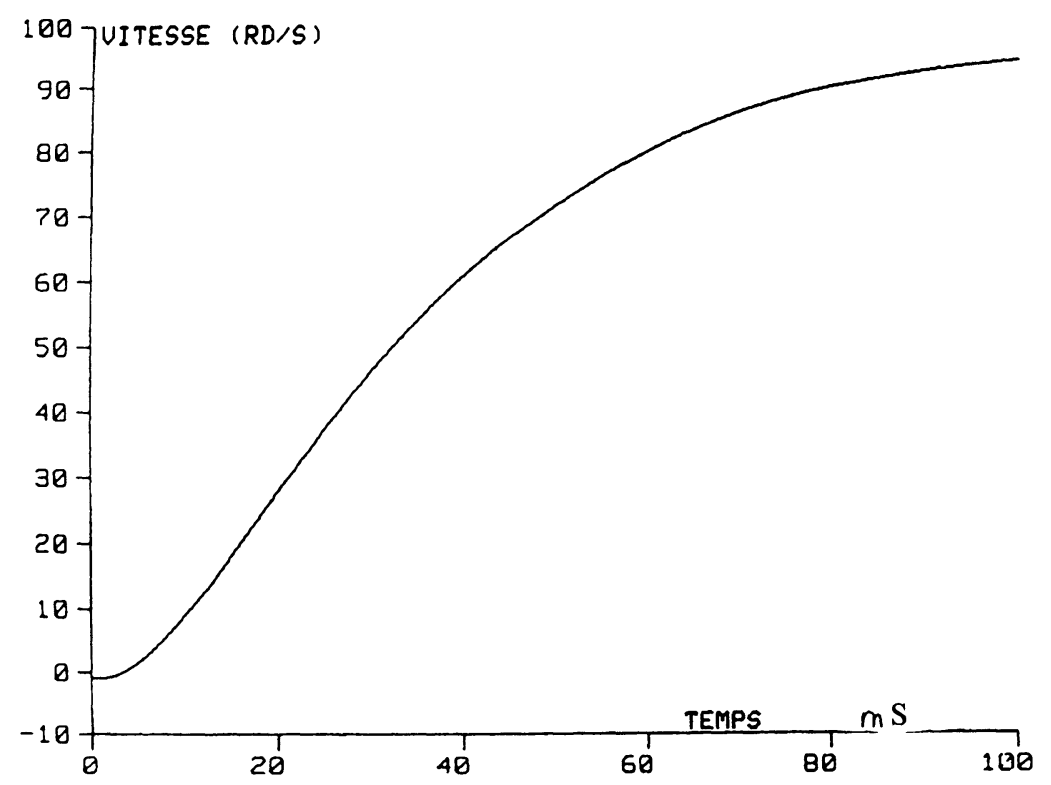

Fig. 5. - Commande non linéaire de vitesse. Modèle échantillonné. $\Omega=f(t)$.

[Speed non linear control. Sampled model. $\Omega=f(t)$.] 


$$
\frac{\mathrm{d} y_{1}}{\mathrm{~d} t}=\alpha f_{1}+\beta f_{2}+\left[\begin{array}{ll}
\alpha g_{1} & \beta g_{2}
\end{array}\right]\left[\begin{array}{l}
V_{\mathrm{d}} \\
V_{\mathrm{q}}
\end{array}\right] \text {. }
$$

Quant à la deuxième sortie, il est cette fois nécessaire de la dériver trois fois pour voir apparaître $V_{\mathrm{d}}$ ou $V_{\mathrm{q}}$ :

$$
\begin{aligned}
\frac{\mathrm{d}^{3} y_{2}}{\mathrm{~d} t^{3}}=f_{1} b_{1} x_{2}+ & f_{2}\left(b_{1} x_{1}+b_{2}\right)+f_{3} b_{0}+ \\
& +\left[\begin{array}{lll}
g_{1} b_{1} x_{2} & g_{2}\left(b_{1} x_{1}+b_{2}\right)
\end{array}\right]\left[\begin{array}{l}
V_{\mathrm{d}} \\
V_{\mathrm{q}}
\end{array}\right] .
\end{aligned}
$$

Nous pouvons ainsi écrire l'équation différentielle :

$$
\left[\begin{array}{c}
\frac{\mathrm{d} y_{1}}{\mathrm{~d} t} \\
\frac{\mathrm{d}^{3} y_{2}}{\mathrm{~d} t^{3}}
\end{array}\right]=B_{0}+A_{0}\left[\begin{array}{c}
V_{\mathrm{d}} \\
V_{\mathrm{q}}
\end{array}\right]
$$

l'expression de $A_{0}$ et $B_{0}$ étant définie dans le paragraphe 3.

Si le déterminant de $A_{0}$ est non nul, ce dont nous avons discuté plus haut, nous pouvons appliquer la commande :

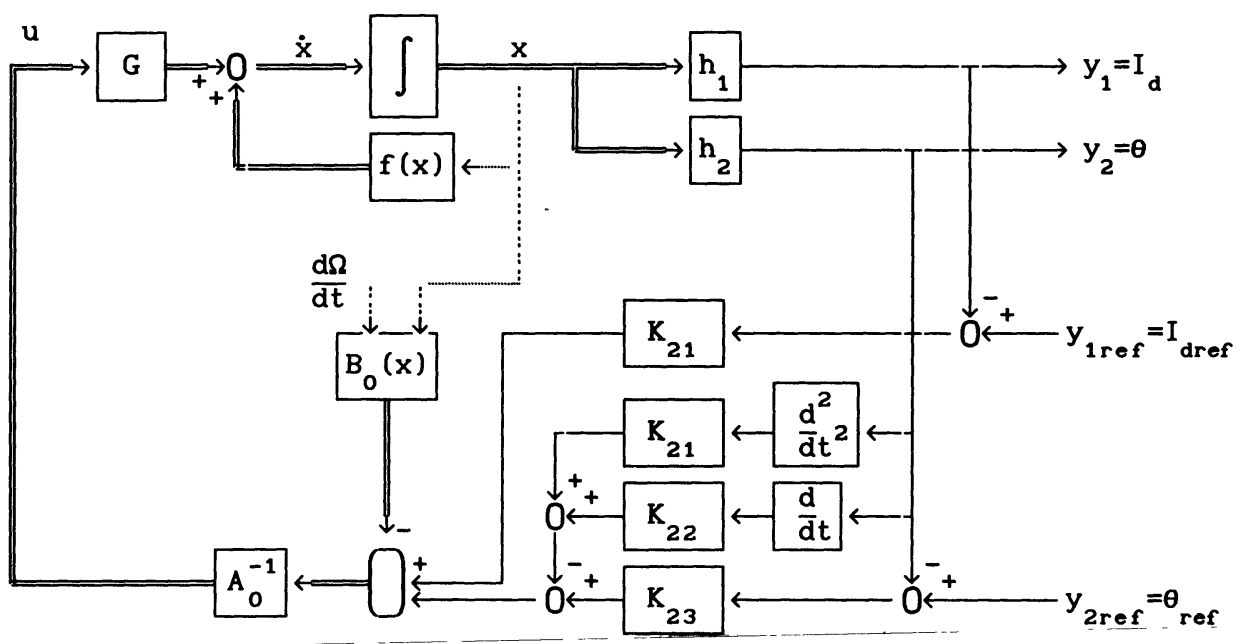

Fig. 6. - Schéma de principe de la commande non linéaire en position.

[Scheme of the position non linear control.]

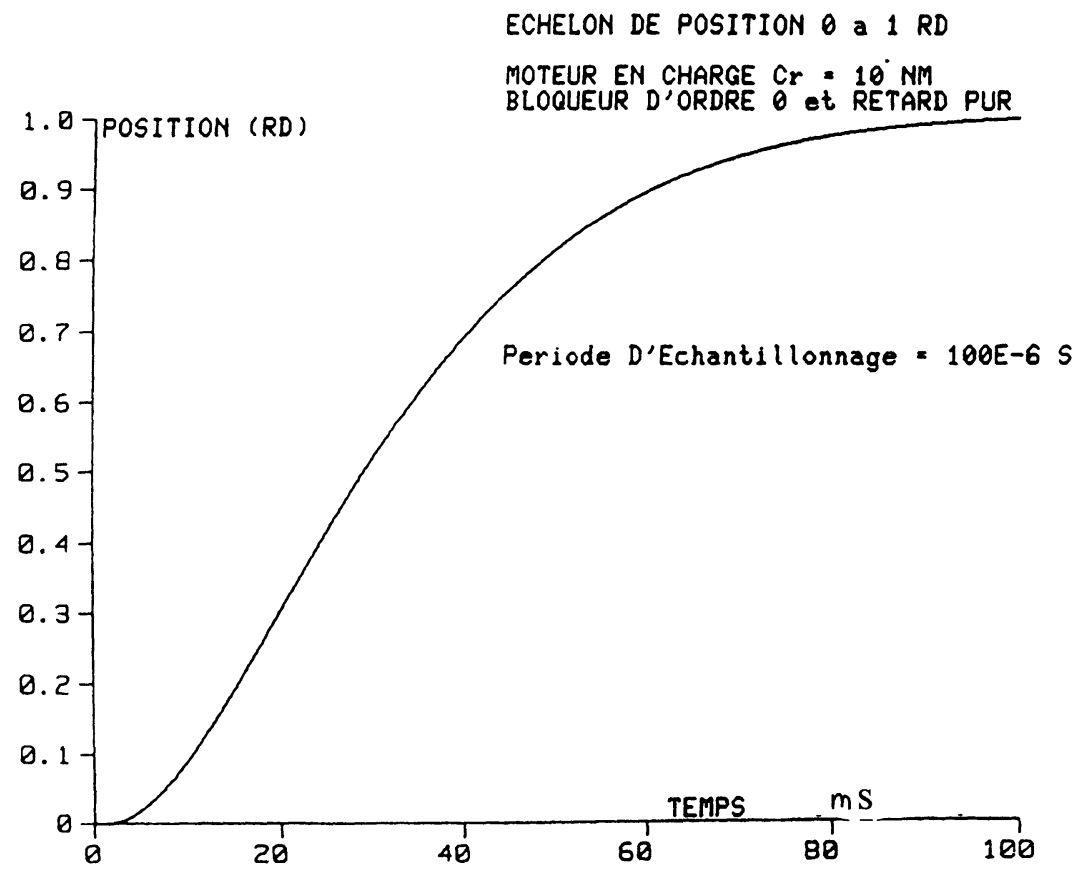

Fig. 7. - Commande non linéaire de position. Modèle échantillonné. $\theta=f(t)$.

[Position non linear control. Sampled model. $\theta=f(t)$.] 
$\left[\begin{array}{c}V_{\mathrm{d}} \\ V_{\mathrm{q}}\end{array}\right]=A_{0}^{-1} \times$

$\times\left\{-B_{0}+\left[\begin{array}{c}K_{11}\left(y_{1 \mathrm{ref}}-y_{1}\right) \\ -K_{21} \frac{\mathrm{d}^{2} y_{2}}{\mathrm{~d} t^{2}}-K_{22} \frac{\mathrm{d} y_{2}}{\mathrm{~d} t}+K_{23}\left(y_{2 \mathrm{ref}}-y_{2}\right)\end{array}\right]\right\}$
Ceci nous permet d'imposer le régime statique $y_{1}=y_{1 \text { ref }}$ et $y_{2}=y_{2 \text { ref }}$, avec une dynamique sur l'erreur régie par une équation du type :

$$
\begin{gathered}
\frac{\mathrm{d} e_{1}}{\mathrm{~d} t}=K_{11} e_{1} \\
\frac{\mathrm{d}^{3} e_{2}}{\mathrm{~d} t^{3}}=-K_{21} \frac{\mathrm{d}^{2} e_{2}}{\mathrm{~d} t^{2}}-K_{22} \frac{\mathrm{d} e_{2}}{\mathrm{~d} t}+K_{23} e_{2} \\
\text { avec } K_{11}, K_{21}, K_{22} \text { et } K_{23} \in \mathbb{R}^{+}
\end{gathered}
$$

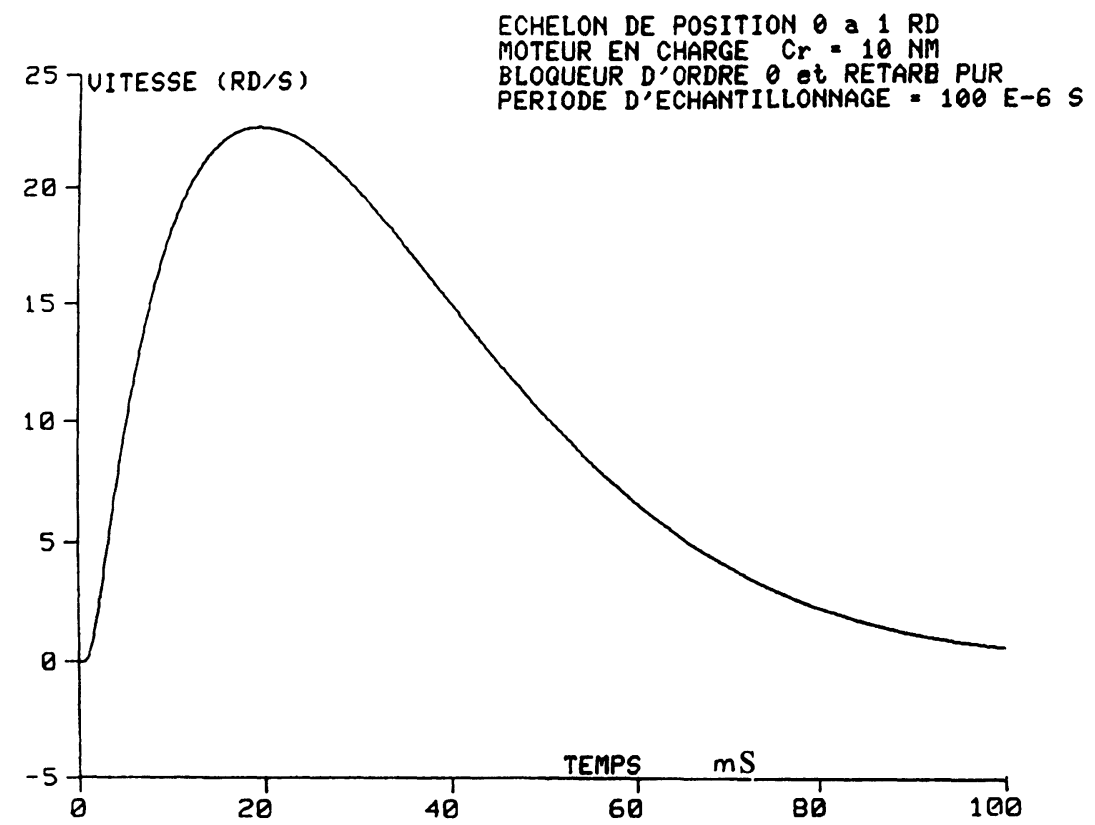

Fig. 8. - Commande non linéaire de position. Modèle échantillonné. $\Omega=f(t)$

[Position non linear control. Sampled model. $\Omega=f(t)$.]

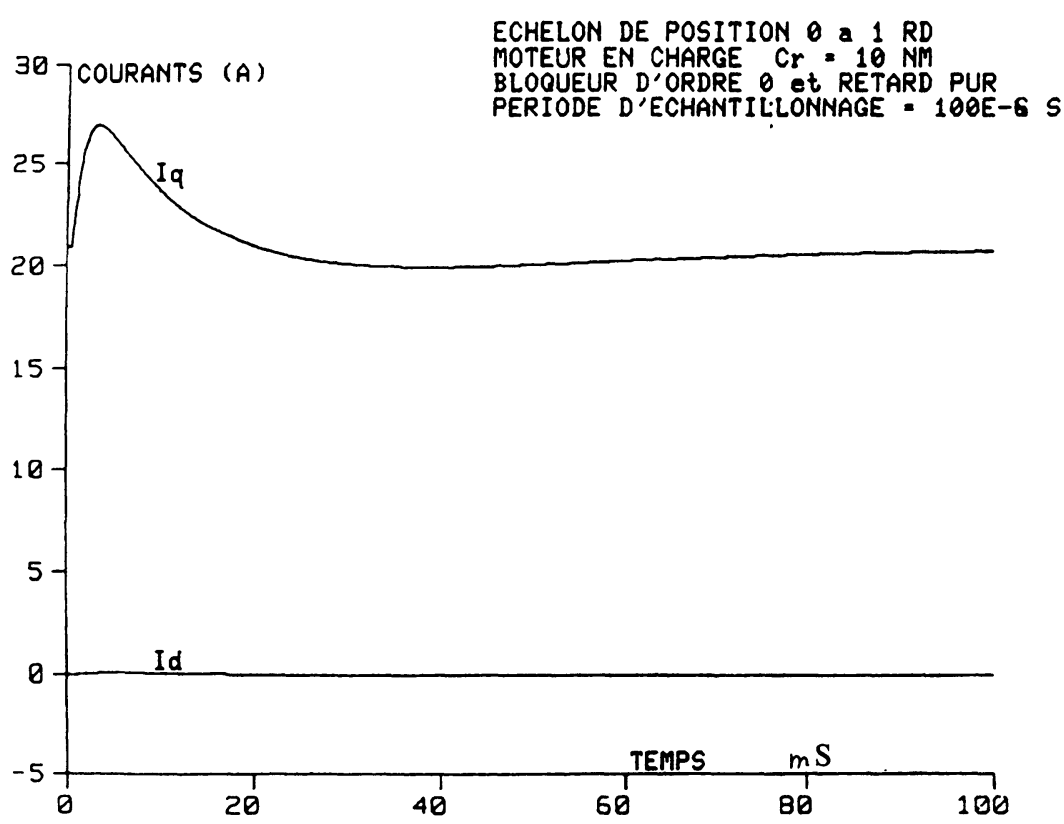

Fig. 9. - Commande non linéaire de position. Modèle échantillonné. $I_{\mathrm{d}}=f(t)$ et $I_{\mathrm{q}}=f(t)$.

[Position non linear control. Sampled model. $I_{\mathrm{d}}=f(t)$ and $I_{\mathrm{q}}=f(t)$.] 
et

$$
e_{1}=y_{1 \text { ref }}-y_{1} \quad e_{2}=y_{2 \text { ref }}-y_{2}
$$

(ce qui revient à imposer le comportement statique et dynamique du courant $i_{\mathrm{d}}$ et de la position $\theta$ ). Il faut remarquer que l'algorithme d'asservissement en position est analogue à l'algorithme d'asservissement de la vitesse, ce qui simplifie grandement l'implantation.

Les figures 7, 8 et 9 montrent un essai indiciel sur la position du moteur, utilisant l'algorithme venant d'être exposé. Le moteur est en charge (charge de $10 \mathrm{Nm}$ ). Le temps de réponse est approximativement de $90 \mathrm{~ms}$, pour un échelon de $1 \mathrm{rad}$ et pour un courant ne dépassant pas le courant maximal admissible par la machine (30 A). Cette simulation prend en compte les bloqueurs d'ordre 0 et le temps de retard dû au calcul. Nous pouvons voir que la régulation réagit particulièrement bien.

\section{Conclusion.}

Nous avons présenté ici des algorithmes non linéaires de régulations de vitesse ou de position d'une machine synchrone autopilotée.

Contrairement aux régulateurs classiques, ces algorithmes prennent en compte les non-linéarités de notre système, celui-ci réagira donc de manière équivalente aux sollicitations demandant de grandes variations (de vitesse ou de position), et aux sollicitations demandant de petites variations. De plus ces régulations restent valables lorsque les dynamiques de la vitesse et des courants ne sont pas suffisamment éloignées pour que l'on justifie leur découplage.

Nous avons testé ces algorithmes en tenant compte des problèmes dus à la quantification (blo- quage et temps de retard dû au temps de calcul). Nous avons vu que le comportement du système reste tout à fait acceptable pour des périodes d'échantillonnage très accessibles à un système utilisant un processeur de signal classique.

Nous avons également pu nous rendre compte qu'il était aisé de passer d'une régulation non linéaire de vitesse à une régulation non linéaire de position. Un même système pourra donc facilement être conçu pour pouvoir fonctionner dans ces deux modes, en utilisant presque la même structure. Le but que nous nous sommes maintenant fixé est d'atténuer les problèmes dus à la discrétisation de notre système grâce à des algorithmes non linéaires intégrant prédicteur et bloqueurs améliorés, de manière à améliorer les performances de notre actionneur électrique.

\section{Annexe.}

\section{Caractéristiques du moteur :}

Courant nominal $i=20 \mathrm{~A}$; Vitesse nominale $=$ $2200 \mathrm{tr} / \mathrm{min}$;

Courant transitoire maximal $i_{\max }=30 \mathrm{~A}$;

Couple maximal transitoire $=14 \mathrm{Nm}$; Couple permanent maximum à l'arrêt $=10 \mathrm{Nm}$;

Couple maximum à $2200 \mathrm{tr} / \mathrm{min}=8,5 \mathrm{Nm}$;

$R_{\mathrm{s}}=0,6 \Omega, L_{\mathrm{d}}=1,4 \mathrm{mH}, \quad L_{\mathrm{q}}=2,8 \mathrm{mH}, p=4$, $\Phi_{\mathrm{f}}=0,12 \mathrm{~Wb}$.

A partir de ces valeurs nous pouvons calculer la valeur du courant de désexcitation : $i_{\mathrm{d}}=85 \mathrm{~A}$.

\section{Remerciements.}

Les auteurs tiennent à remercier D. Normand-Cyrot et $\mathrm{S}$. Monaco pour l'aide apportée à l'accomplissement de ce travail.

\section{Bibliographie}

[1] Byrnes C. and Isidori A., A frequency domain philosophy for non linear systems, 23th IEEE Conf. decision and control (1984).

[2] De Luca A., Ulivi G., Full linearization of induction motors, Non linear state feedback, Proc. of the 26th conference on decision and control, Los Angeles CA (December 1987) pp. 1765-1770.

[3] Georgiou G., Adaptative Feedback Linearization and tracking for induction motors, IFAC, Workshop on evaluation of adaptative control strategies in industrial applications, Tbilisi, USSR (October 1989).

[4] IsIDORI A., Non linear control systems, an introduction, 2nd edition (Springer-Verlag).

[5] Louis J. P. et Le Pioufle B., Représentation fonctionnelle des machines à courant alternatif et de leur alimentation pour leur commande en vitesse variable, Journées S.E.E., Les actionneurs électriques, esclaves des temps modernes (9 et 10 novembre 1989).
[6] Louis J. P., Multon B., Lavabre M., Commande des moteurs à courant continu à vitesse variable, Techniques de l'Ingénieur (décembre 1988).

[7] Monaco S., NoRMAND-CYRot D., Sur la commande digitale d'un système non linéaire à déphasage minimal, Proc., 8th Int. Conf. on Analys. and Optim. of Systems (Antibes 1988).

[8] Monaco S., Normand-Cyrot D., The immersion under feedback of a multidimensional discrete time non linear system into a linear system, Int. J. Control 38 (1983) 245-261.

[9] Monaco S., Normand-Cyrot D. et Stornelli S., On the linearizing feedback in nonlinear sampled data control schemes, Proc. of the IEEE Conf. on Decision and Control, Athenes (1983) pp. 2056-2060.

[10] LeOnhard W., Control of electrical drives (Springer-Verlag) 1985. 